\title{
HIGHER MINORS AND VAN KAMPEN'S OBSTRUCTION
}

\author{
ERAN NEVO
}

\begin{abstract}
We generalize the notion of graph minors to all (finite) simplicial complexes. For every two simplicial complexes $H$ and $K$ and every nonnegative integer $m$, we prove that if $H$ is a minor of $K$ then the non vanishing of Van Kampen's obstruction in dimension $m$ (a characteristic class indicating non embeddability in the $(m-1)$-sphere) for $H$ implies its non vanishing for $K$. As a corollary, based on results by Van Kampen [19] and Flores [4], if $K$ has the $d$-skeleton of the $(2 d+2)$-simplex as a minor, then $K$ is not embeddable in the $2 d$-sphere.

We answer affirmatively a problem asked by Dey et. al. [2] concerning topology-preserving edge contractions, and conclude from it the validity of the generalized lower bound inequalities for a special class of triangulated spheres.
\end{abstract}

\section{Introduction}

The concept of graph minors has proved be to very fruitful. A famous result by Kuratowski asserts that a graph can be embedded into a 2-sphere if and only if it contains neither of the graphs $K_{5}$ and $K_{3,3}$ as minors. We wish to generalize the notion of graph minors to all (finite) simplicial complexes in a way that would produce analogous statements for embeddability of higher dimensional complexes in higher dimensional spheres. We hope that these higher minors will be of interest in future research, and indicate some results and problems to support this hope.

Let $K$ and $K^{\prime}$ be simplicial complexes. $K \mapsto K^{\prime}$ is called a deletion if $K^{\prime}$ is a subcomplex of $K . K \mapsto K^{\prime}$ is called an admissible contraction if $K^{\prime}$ is obtained from $K$ by identifying two distinct vertices of $K, v$ and $u$, such that $v$ and $u$ are not contained in any missing face of $K$ of dimension $\leq \operatorname{dim}(K)$. (A set $T$ is called a missing face of $K$ if it is not an element of $K$ while all its proper subsets are.) Specifically, $K^{\prime}=\{T: u \notin T \in K\} \cup\{(T \backslash\{u\}) \cup\{v\}: u \in T \in$ $K\}$. An equivalent formulation of the condition for admissible contractions is that the following holds:

$$
\operatorname{skel}_{\operatorname{dim}(K)-2}(\operatorname{lk}(v, K) \cap \operatorname{lk}(u, K))=\operatorname{lk}(\{v, u\}, K)
$$

Received May 5, 2006. 
where $\operatorname{skel}_{m}(K)$ is the subcomplex of $K$ consisting of faces of dimension $\leq m$ and $\operatorname{lk}(T, K)=\{F \in K: T \cap F=\emptyset, F \cup T \in K\}$ is the link of $T$ in $K$ for a face $T \in K$. For $K$ a graph, (1) just means that $\{v, u\}$ is an edge in $K$.

We say that a simplicial complex $H$ is a minor of $K$, and denote it by $H<K$, if $H$ can be obtained from $K$ by a sequence of admissible contractions and deletions (the relation $<$ is a partial order). Note that for graphs this is the usual notion of a minor.

Remarks. (1) In equation (1), the restriction to the skeleton of dimension at most $\operatorname{dim}(K)-2$ can be replaced by restriction to the skeleton of dimension at $\operatorname{most} \min \{\operatorname{dim}(\operatorname{lk}(u, K)), \operatorname{dim}(\operatorname{lk}(v, K))\}-1$, making the condition for admissible contraction local, and weaker. All the results and proofs in this paper hold verbatim for this notion of a minor as well.

(2) In the definition of a minor, without loss of generality we may replace the local condition from the remark above by the following stronger local condition, called the Link Condition for $\{u, v\}$ :

$$
\operatorname{lk}(u, K) \cap \operatorname{lk}(v, K)=\operatorname{lk}(\{u, v\}, K) .
$$

To see this, let $K \mapsto K^{\prime}$ be an admissible contraction which is obtained by identifying the vertices $u$ and $v$ where $\operatorname{dim}(\operatorname{lk}(u, K)) \leq \operatorname{dim}(\operatorname{lk}(v, K))$. Delete from $K$ all the faces $F \uplus\{u\}$ such that $F \uplus\{u, v\}$ is a missing face of dimension $\operatorname{dim}(\operatorname{lk}(u, K))+2$, to obtain a simplicial complex $L$. Note that $\{u, v\}$ satisfies the Link Condition in $L$, and the identification of $u$ with $v$ in $L$ results in $K^{\prime}$. I thank an anonymous referee for this remark.

We now relate this minor notion to Van Kampen's obstruction in cohomology; following Sarkaria [14] we will work with deleted joins and with $Z_{2}$ coefficients (background and definitions appear in the next section).

Theorem 1.1. Let $\operatorname{Sm}^{m}(L) \in H_{S}^{m}\left(L_{*}, \mathrm{Z}_{2}\right)$ denote Van Kampen's obstruction (in equivariant cohomology) for a simplicial complex $L$, where $L_{*}$ is the deleted join of $L$. Let $H$ and $K$ be simplicial complexes. If $H<K$ and $\operatorname{Sm}^{m}(H) \neq 0$ then $\operatorname{Sm}^{m}(K) \neq 0$.

For any positive integer $d$ let $H(d)$ be the $(d-1)$-skeleton of the $2 d$ dimensional simplex. A well known result by Van Kampen and Flores [4], [19] asserts that the Van Kampen obstruction of $H(d)$ in dimension $(2 d-1)$ does not vanish, and hence $H(d)$ is not embeddable in the 2(d-1)-sphere (note that the case $H(2)=K_{5}$ is part of the easier direction of Kuratowski's theorem).

Corollary 1.2. For every $d \geq 1$, if $H(d)<K$ then $K$ is not embeddable in the $2(d-1)$-sphere. 
Remark. Corollary 1.2 would also follow from the following conjecture:

Conjecture 1.3. If $H<K$ and $K$ is embeddable in the $m$-sphere then $H$ is embeddable in the $m$-sphere.

The following theorem answers in the affirmative a question asked by Dey et. al. [2], who already proved the dimension $\leq 3$ case.

THEOREM 1.4. Given an edge in a triangulation of a compact PL (piecewise linear)-manifold without boundary, its contraction results in a PL-homeomorphic space if and only if it satisfies the Link Condition (2).

In Section 2 we give the needed background on Van Kampen's obstruction and Smith characteristic class. In Section 3 we prove Theorem 1.1 and show some applications. In Section 4 we prove an analogue of Theorem 1.1 for deleted products and $Z$ coefficients. In Section 5 we prove Theorem 1.4 and deduce from it some $f$-vector consequences. In Section 6 we compare higher minors with graph minors.

\section{Algebraic-topological background}

The presentation here is based on work of Sarkaria [14], [15] who attributes it to $\mathrm{Wu}$ [22] and all the way back to Van Kampen [19]. It is a Smith theoretic interpretation of Van Kampen's obstructions.

Let $K$ be a simplicial complex. The join $K * K$ is the simplicial complex $\left\{S^{1} \uplus T^{2}: S, T \in K\right\}$ (the superscripts indicate two disjoint copies of $K$ ). The deleted join $K_{*}$ is the subcomplex $\left\{S^{1} \uplus T^{2}: S, T \in K, S \cap T=\emptyset\right\}$. The restriction of the involution $\tau: K * K \rightarrow K * K, \tau\left(S^{1} \cup T^{2}\right)=T^{1} \cup S^{2}$ to $K_{*}$ is into $K_{*}$. It induces a $Z_{2}$-action on the cochain complex $C^{*}\left(K_{*} ; Z_{2}\right)$. For a simplicial cochain complex $C$ over $Z_{2}$ with a $Z_{2}$-action $\tau$, let $C_{S}$ be its subcomplex of symmetric cochains, $\{c \in C: \tau(c)=c\}$. Restriction induces an action of $\tau$ as the identity map on $C_{S}$. Note that the following sequence is exact in dimensions $\geq 0$ :

$$
0 \longrightarrow C_{S}\left(K_{*}\right) \longrightarrow C\left(K_{*}\right) \stackrel{\mathrm{id}+\tau}{\longrightarrow} C_{S}\left(K_{*}\right) \longrightarrow 0
$$

where $C_{S}\left(K_{*}\right) \rightarrow C\left(K_{*}\right)$ is the trivial injection. (The only part of this statement that may be untrue for a non-free simplicial cochain complex $C$ over $Z_{2}$ with a $Z_{2}$-action $\tau$, is that id $+\tau$ is surjective.) Thus, there is an induced long exact sequence in cohomology

$$
\begin{aligned}
H_{S}^{0}\left(K_{*}\right) \stackrel{\mathrm{Sm}}{\longrightarrow} H_{S}^{1}\left(K_{*}\right) & \longrightarrow \cdots \longrightarrow H_{S}^{q}\left(K_{*}\right) \\
& \longrightarrow H^{q}\left(K_{*}\right) \longrightarrow H_{S}^{q}\left(K_{*}\right) \stackrel{\mathrm{Sm}}{\longrightarrow} H_{S}^{q+1}\left(K_{*}\right) \longrightarrow \cdots
\end{aligned}
$$


Composing the connecting homomorphism $\mathrm{Sm} m$ times we obtain a map $\mathrm{Sm}^{m}: H_{S}^{0}\left(K_{*}\right) \rightarrow H_{S}^{m}\left(K_{*}\right)$. For the fundamental 0-cocycle $1_{K_{*}}$, i.e. the one which maps $\sum_{v \in\left(K_{*}\right)_{0}} a_{v} v \mapsto \sum_{v \in\left(K_{*}\right)_{0}} a_{v} \in \mathrm{Z}_{2}$, let $\left[1_{K_{*}}\right]$ denotes its image in $H_{S}^{0}\left(K_{*}\right) . \mathrm{Sm}^{m}\left(\left[1_{K_{*}}\right]\right)$ is called the $m$-th Smith characteristic class of $K_{*}$, denoted also as $\operatorname{Sm}^{m}(K)$.

Theorem 2.1 (Sarkaria [15] Theorem 6.5, see also Wu [22] pp. 114-118). For every $d \geq 1, \operatorname{Sm}^{2 d-1}\left(1_{H(d)_{*}}\right) \neq 0$.

THeORem 2.2 (Sarkaria [15] Theorem 6.4 and [14] p. 6). If a simplicial complex $K$ embeds in $\mathrm{R}^{m}$ (or in the $m$-sphere) then $\mathrm{Sm}^{m+1}\left(1_{K_{*}}\right)=0$.

SkETCH OF PROOF. The definition of Smith class makes sense for singular homology as well; the obvious map from the simplicial chain complex to the singular one induces an isomorphism between the corresponding Smith classes. The definition of deleted join makes sense for subspaces of a Euclidean space as well (see e.g. [12], 5.5); thus an embedding $|K|$ of $K$ into $\mathrm{R}^{m}$ induces a continuous $\mathrm{Z}_{2}$-map from $|K|_{*}$ into the join of $\mathrm{R}^{m}$ with itself minus the diagonal, which is $\mathrm{Z}_{2}$-homotopic to the antipodal $m$-sphere, $S^{m}$. The equivariant cohomology of $S^{m}$ over $\mathrm{Z}_{2}$ is isomorphic to the ordinary cohomology of $\mathrm{R} P^{m}$ over $Z_{2}$, which vanishes in dimension $m+1$. We get that $\operatorname{Sm}^{m+1}\left(S^{m}\right)$ maps to $\mathrm{Sm}^{m+1}\left(1_{|K|_{*}}\right)$ and hence the later equals to zero as well. But $\left|K_{*}\right|$ and $|K|_{*}$ are $\mathrm{Z}_{2}$-homotopic, hence $\mathrm{Sm}^{m+1}\left(1_{K_{*}}\right)=0$.

\section{A proof of Theorem 1.1}

The idea is to define an injective chain map $\phi: C_{*}\left(H ; \mathrm{Z}_{2}\right) \rightarrow C_{*}\left(K ; \mathbf{Z}_{2}\right)$ which induces $\phi\left(\operatorname{Sm}^{m}\left(1_{K_{*}}\right)\right)=\operatorname{Sm}^{m}\left(1_{H_{*}}\right)$ for every $m \geq 0$.

Lemma 3.1. Let $K \mapsto K^{\prime}$ be an admissible contraction. Then it induces an injective chain map $\phi: C_{*}\left(K^{\prime} ; \mathrm{Z}_{2}\right) \rightarrow C_{*}\left(K ; \mathrm{Z}_{2}\right)$.

Proof. Fix a labeling of the vertices of $K, v_{0}, v_{1}, \ldots, v_{n}$, such that $K^{\prime}$ is obtained from $K$ by identifying $v_{0} \mapsto v_{1}$ where $\operatorname{dim}\left(\operatorname{lk}\left(v_{0}, K\right)\right) \leq \operatorname{dim}\left(\operatorname{lk}\left(v_{1}, K\right)\right)$.

Let $F \in K^{\prime}$. If $F \in K$, define $\phi(F)=F$. If $F \notin K$, define $\phi(F)=$ $\sum\left\{(F \backslash v) \cup v_{0}: v \in F,(F \backslash v) \cup v_{0} \in K\right\}$. Note that if $F \notin K$ then $v_{1} \in F$ and $\left(F \backslash v_{1}\right) \cup v_{0} \in K$, so the sum above is nonzero. Extend linearly to obtain a map $\phi: C_{*}\left(K^{\prime} ; \mathrm{Z}_{2}\right) \rightarrow C_{*}\left(K ; \mathrm{Z}_{2}\right)$.

First, let us check that $\phi$ is a chain map, i.e. that it commutes with the boundary maps $\partial$. It is enough to verify this for the basis elements $F$ where $F \in K^{\prime}$. If $F \in K$ then $\operatorname{supp}(\partial F) \subseteq K$, hence $\partial(\phi F)=\partial F=\phi(\partial F)$. If $F \notin K$ then $\partial(\phi F)=\partial\left(\sum\left\{(F \backslash v) \cup v_{0}: v \in F,(F \backslash v) \cup v_{0} \in K\right\}\right)$, and as 
we work over $Z_{2}$, this equals

$$
\begin{aligned}
& \partial(\phi F)=\sum\left\{F \backslash v: v \in F,(F \backslash v) \cup v_{0} \in K\right\} \\
& +\sum\left\{(F \backslash\{u, v\}) \cup v_{0}: u, v \in F,(F \backslash v) \cup v_{0} \in K,(F \backslash u) \cup v_{0} \notin K\right\} .
\end{aligned}
$$

On the other hand $\phi(\partial F)=\phi\left(\sum\{F \backslash u: u \in F, F \backslash u \in K\}\right)+\phi\left(\sum\{F \backslash u\right.$ : $u \in F, F \backslash u \notin K\})$ and as we work over $Z_{2}$, this equals

$$
\begin{array}{r}
\phi(\partial F)=\sum\{F \backslash u: u \in F,(F \backslash u) \in K\}+\sum\left\{(F \backslash\{u, v\}) \cup v_{0}: u,\right. \\
\left.v \in F,(F \backslash\{u, v\}) \cup v_{0} \in K,(F \backslash v) \in K,(F \backslash u) \notin K\right\} .
\end{array}
$$

It suffices to show that in equations (3) and (4) the left summands on the RHSs are equal, as well as the right summands on the RHSs. This follows from observation 3.2 below. Thus $\phi$ is a chain map.

Second, let us check that $\phi$ is injective. Let $\pi_{K}$ be the restriction map $C_{*}\left(K^{\prime} ; \mathbf{Z}_{2}\right) \rightarrow \oplus\left\{Z_{2} F: F \in K^{\prime} \cap K\right\}, \pi_{K}\left(\sum\left\{\alpha_{F} F: F \in K^{\prime}\right\}\right)=\sum\left\{\alpha_{F} F:\right.$ $\left.F \in K^{\prime} \cap K\right\}$. Similarly, let $\pi_{K}^{\perp}$ be the restriction map $C_{*}\left(K^{\prime} ; \mathbf{Z}_{2}\right) \rightarrow \oplus\left\{\mathbf{Z}_{2} F\right.$ : $\left.F \in K^{\prime} \backslash K\right\}$. Note that for a chain $c \in C_{*}\left(K^{\prime} ; Z_{2}\right), c=\pi_{K}(c)+\pi_{K}^{\perp}(c)$ and $\operatorname{supp}\left(\phi\left(\pi_{K}(c)\right)\right) \cap \operatorname{supp}\left(\phi\left(\pi_{K}^{\perp}(c)\right)\right)=\emptyset$. Assume that $c_{1}, c_{2} \in C_{*}\left(K^{\prime} ; \mathbf{Z}_{2}\right)$ such that $\phi\left(c_{1}\right)=\phi\left(c_{2}\right)$. Then $\pi_{K}\left(c_{1}\right)=\phi\left(\pi_{K}\left(c_{1}\right)\right)=\phi\left(\pi_{K}\left(c_{2}\right)\right)=\pi_{K}\left(c_{2}\right)$, and $\phi\left(\pi_{K}^{\perp}\left(c_{1}\right)\right)=\phi\left(\pi_{K}^{\perp}\left(c_{2}\right)\right)$. Note that if $F_{1}, F_{2} \notin K$ then $F_{1}, F_{2} \in K^{\prime}$ and if $F_{1} \neq F_{2}$ then $\operatorname{supp}\left(\phi\left(1 F_{1}\right)\right) \ni\left(F_{1} \backslash v_{1}\right) \cup v_{0} \notin \operatorname{supp}\left(\phi\left(1 F_{2}\right)\right)$. Hence also $\pi_{K}^{\perp}\left(c_{1}\right)=\pi_{K}^{\perp}\left(c_{2}\right)$. Thus $c_{1}=c_{2}$.

OBSERVATION 3.2. Let $K \mapsto K^{\prime}, v_{0} \mapsto v_{1}$ be an admissible contraction with $\operatorname{dim}\left(\operatorname{lk}\left(v_{0}, K\right)\right) \leq \operatorname{dim}\left(\operatorname{lk}\left(v_{1}, K\right)\right)$. Let $K^{\prime} \ni F \notin K$ and $v \in F$. Then $(F \backslash v) \in K$ if and only if $(F \backslash v) \cup v_{0} \in K$.

Proof. Assume $F \backslash v \in K$. As $\left(F \backslash v_{1}\right) \cup v_{0} \in K$ we only need to check the case $v \neq v_{1}$. We proceed by induction on $\operatorname{dim}(F)$. As $\left\{v_{0}, v_{1}\right\} \in K$ whenever $\operatorname{dim}(K)>0$ (and whenever $\operatorname{dim}\left(\operatorname{lk}\left(v_{0}, K\right)\right) \geq 0$, if we use the weaker local condition for admissible contractions), the case $\operatorname{dim}(F) \leq 1$ is clear. (If $\operatorname{dim}(K)=0$ there is nothing to prove. For the weaker local condition for admissible contractions, if $\left.\operatorname{lk}\left(v_{0}, K\right)\right)=\emptyset$ then there is nothing to prove.) By the induction hypothesis we may assume that all the proper subsets of $(F \backslash v) \cup v_{0}$ are in $K$. Also $v_{0}, v_{1} \in(F \backslash v) \cup v_{0}$. The admissibility of the contraction implies that $(F \backslash v) \cup v_{0} \in K$. The other direction is trivial.

Lemma 3.3. Let $\phi: C_{*}\left(K^{\prime} ; \mathrm{Z}_{2}\right) \rightarrow C_{*}\left(K ; \mathrm{Z}_{2}\right)$ be the injective chain map defined in the proof of Lemma 3.1 for an admissible contraction $K \mapsto K^{\prime}$. Then for every $m \geq 0, \phi^{*}\left(\operatorname{Sm}^{m}\left(\left[1_{K_{*}}\right]\right)\right)=\operatorname{Sm}^{m}\left(\left[1_{K_{*}^{\prime}}\right]\right)$ for the induced map $\phi^{*}$. 
Proof. For two simplicial complexes $L$ and $L^{\prime}$ and a field $k$, the following map is an isomorphism of chain complexes:

$$
\begin{gathered}
\alpha=\alpha_{L, L^{\prime}, k}: C(L ; k) \otimes_{k} C\left(L^{\prime} ; k\right) \longrightarrow C\left(L * L^{\prime} ; k\right), \\
\alpha\left((1 T) \otimes\left(1 T^{\prime}\right)\right)=1\left(T \uplus T^{\prime}\right)
\end{gathered}
$$

where $T \in L, T^{\prime} \in L^{\prime}$ and $\alpha$ is extended linearly. In case $L=L^{\prime}$ (in the definition of join we think of $L$ and $L^{\prime}$ as two disjoint copies of $L$ ) and $k$ is understood we denote $\alpha_{L, L^{\prime}, k}=\alpha_{L}$.

Thus there is an induced chain map $\phi_{*}: C_{*}\left(K^{\prime} * K^{\prime} ; \mathbf{Z}_{2}\right) \rightarrow C_{*}\left(K * K ; \mathbf{Z}_{2}\right)$, $\phi_{*}=\alpha_{K} \circ \phi \otimes \phi \circ \alpha_{K^{\prime}}^{-1}$ where $\phi \otimes \phi: C\left(K^{\prime} ; \mathrm{Z}_{2}\right) \otimes \mathrm{z}_{2} C\left(K^{\prime} ; \mathrm{Z}_{2}\right) \rightarrow C\left(K ; \mathrm{Z}_{2}\right) \otimes \mathrm{z}_{2}$ $C\left(K ; \mathbf{Z}_{2}\right)$ is defined by $\phi \otimes \phi\left(c \otimes c^{\prime}\right)=\phi(c) \otimes \phi\left(c^{\prime}\right)$ (which this is a chain map).

Consider the subcomplex $C_{*}\left(K_{*}^{\prime} ; \mathrm{Z}_{2}\right) \subseteq C_{*}\left(K^{\prime} * K^{\prime} ; \mathrm{Z}_{2}\right)$. We now verify that every $c \in C_{*}\left(K_{*}^{\prime} ; \mathbf{Z}_{2}\right)$ satisfies $\phi_{*}(c) \in C_{*}\left(K_{*} ; \mathbf{Z}_{2}\right)$. It is enough to check this for chains of the form $c=1\left(S^{1} \cup T^{2}\right)$ where $S, T \in K^{\prime}$ and $S \cap T=\emptyset$. For a collection of sets $A$ let $V(A)=\cup_{a \in A} a$. Clearly if the condition

$$
V(\operatorname{supp}(\phi(S))) \cap V(\operatorname{supp}(\phi(T)))=\emptyset
$$

is satisfied then we are done. If $v_{1} \notin S, v_{1} \notin T$, then $\phi(S)=S, \phi(T)=T$ and (5) holds. If $T \ni v_{1} \notin S$, then $\phi(S)=S$ and $V(\operatorname{supp} \phi(T)) \subseteq T \cup\left\{v_{0}\right\}$. As $v_{0} \notin S$ condition (5) holds. By symmetry, (5) holds when $S \ni v_{1} \notin T$ as well.

With abuse of notation (which we will repeat) we denote the above chain map by $\phi, \phi: C_{*}\left(K_{*}^{\prime} ; \mathbf{Z}_{2}\right) \rightarrow C_{*}\left(K_{*} ; \mathbf{Z}_{2}\right)$. For a simplicial complex $L$, the involution $\tau_{L}: L_{*} \rightarrow L_{*}, \tau_{L}\left(S^{1} \cup T^{2}\right)=T^{1} \cup S^{2}$ induces a $Z_{2}$-action on $C_{*}\left(L_{*} ; Z_{2}\right)$. It is immediate to check that $\alpha_{L, L^{\prime}, k}$ and $\phi \otimes \phi$ commute with these $Z_{2}$-actions, and hence so does their composition, $\phi$. Thus, we have proved that $\phi: C_{*}\left(K_{*}^{\prime} ; \mathbf{Z}_{2}\right) \rightarrow C_{*}\left(K_{*} ; \mathbf{Z}_{2}\right)$ is a $\mathbf{Z}_{2}$-chain map.

Therefore, there is an induced map on the symmetric cohomology rings $\phi$ : $H_{S}^{*}\left(K_{*}\right) \rightarrow H_{S}^{*}\left(K_{*}^{\prime}\right)$ which commutes with the connecting homomorphisms $\mathrm{Sm}: H_{S}^{i}(L) \rightarrow H_{S}^{i+1}(L)$ for $L=K_{*}, K_{*}^{\prime}$.

Let us check that for the fundamental 0 -cocycles $\phi\left(\left[1_{K_{*}}\right]\right)=\left[1_{K_{*}^{\prime}}\right]$ holds. A representing cochain is $1_{K_{*}}: \oplus_{v \in\left(K_{*}\right)_{0}} \mathrm{Z}_{2} v \longrightarrow \mathrm{Z}_{2}, 1_{K_{*}}(1 v) \stackrel{\text {. }}{=} 1$. As $\left.\phi\right|_{C_{0}\left(K_{*}^{\prime}\right)}=i d$ (w.r.t. the obvious injection $\left.\left(K_{*}^{\prime}\right)_{0} \longrightarrow\left(K_{*}\right)_{0}\right)$, for every $u \in\left(K_{*}^{\prime}\right)_{0}\left(\phi 1_{K_{*}}\right)(u)=1_{K_{*}}\left(\left.\phi\right|_{C_{0}\left(K_{*}^{\prime}\right)}(u)\right)=1_{K_{*}}(u)=1$, thus $\phi\left(1_{K_{*}}\right)=1_{K_{*}^{\prime}}$.

As $\phi$ commutes with the Smith connecting homomorphisms, for every $m \geq$ $0, \phi\left(\operatorname{Sm}^{m}\left(1_{K_{*}}\right)\right)=\operatorname{Sm}^{m}\left(1_{K_{*}^{\prime}}\right)$.

THEOREM 3.4. Let $H$ and $K$ be simplicial complexes. If $H<K$ then there exists an injective chain map $\phi: C_{*}\left(H ; \mathrm{Z}_{2}\right) \rightarrow C_{*}\left(K ; \mathrm{Z}_{2}\right)$ which induces $\phi\left(\operatorname{Sm}^{m}\left(1_{K_{*}}\right)\right)=\operatorname{Sm}^{m}\left(1_{H_{*}}\right)$ for every $m \geq 0$. 
Proof. Let the sequence $K=K^{0} \mapsto K^{1} \mapsto \cdots \mapsto K^{t}=H$ demonstrate the fact that $H<K$. If $K^{i} \mapsto K^{i+1}$ is an admissible contraction, then by Lemmas 3.1 and 3.3 it induces an injective chain map $\phi_{i}: C_{*}\left(K^{i+1} ; Z_{2}\right) \rightarrow$ $C_{*}\left(K^{i} ; Z_{2}\right)$ which in turn induces $\phi_{i}\left(\operatorname{Sm}^{m}\left(1_{\left(K^{i}\right)_{*}}\right)\right)=\operatorname{Sm}^{m}\left(1_{\left(K^{i+1}\right)_{*}}\right)$ for every $m \geq 0$. If $K^{i} \mapsto K^{i+1}$ is a deletion - take $\phi_{i}$ to be the map induced by inclusion, to obtain the same conclusions. Thus, the composition $\phi=\phi_{0} \circ \cdots \circ \phi_{t-1}$ : $C_{*}\left(H ; \mathrm{Z}_{2}\right) \rightarrow C_{*}\left(K ; \mathrm{Z}_{2}\right)$ is as desired.

Proof of Theorem 1.1. By Theorem $3.4 \phi\left(\operatorname{Sm}^{m}\left(1_{K_{*}}\right)\right)=\operatorname{Sm}^{m}\left(1_{H_{*}}\right)$. Thus if $\operatorname{Sm}^{m}\left(1_{H_{*}}\right) \neq 0$ then $\operatorname{Sm}^{m}\left(1_{K_{*}}\right) \neq 0$.

REMARK. The conclusion of Theorem 1.1 would fail if we allow arbitrary identifications of vertices. For example, let $K^{\prime}=K_{5}$ and let $K$ be obtained from $K^{\prime}$ by splitting a vertex $w \in K^{\prime}$ into two new vertices $u, v$, and connecting $u$ to a non-empty proper subset of $\operatorname{skel}_{0}\left(K^{\prime}\right) \backslash\{w\}$, denoted by $A$, and connecting $v$ to $\left(\operatorname{skel}_{0}\left(K^{\prime}\right) \backslash\{w\}\right) \backslash A$. As $K$ embeds into the 2-sphere, $\operatorname{Sm}^{3}(K)=0$. By identifying $u$ with $v$ we obtain $K^{\prime}$, but $\operatorname{Sm}^{3}\left(K^{\prime}\right) \neq 0$. To obtain from this example an example where the edge $\{u, v\}$ is present, let $L=\operatorname{cone}(K) \cup\{u, v\}$ (cone $(K)$ is the cone over $K$ ), and let $L^{\prime}$ be the complex obtained form $L$ by identifying $u$ with $v$. Then $\operatorname{Sm}^{4}(L)=0$ while $\operatorname{Sm}^{4}\left(L^{\prime}\right) \neq 0$.

Example 3.5. Let $K$ be the simplicial complex spanned by the following collection of 2-simplices: $\left(\left(\begin{array}{c}{[7]} \\ 3\end{array}\right) \backslash\{127,137,237\}\right) \cup\{128,138,238,178,278$, $378\}$.

$K$ is not a subdivision of $H(3)$, and its geometric realization even does not contain a subspace homeomorphic to $H(3)$ (as there are no 7 points in $|K|$, each with a neighborhood whose boundary contains a subspace which is homeomorphic to $K_{6}$ ). Nevertheless, contraction of the edge 78 is admissible and results in $H(3)$. By Theorem $1.1 K$ has a non-vanishing Van Kampen's obstruction in dimension 5, and hence is not embeddable in the 4-sphere.

ExAmple 3.6. Let $K_{1}$ be a triangulation of $S^{1}$ (the 1-sphere) and let $K_{2}$ be a triangulation of $S^{2}$. Then $K=K_{1} * K_{2}$ is a triangulation of $S^{4}$. Let $T$ be a missing triangle of $K$ and $L=\operatorname{skel}_{2}(K) \cup\{T\}$. Then $L$ does not embed in $\mathrm{R}^{4}$.

Proof. It is known and easy to prove that every 2-sphere may be reduced to the boundary of the tetrahedron by a sequence of admissible contractions in a way that fixes a chosen triangle from the original triangulation (e.g. [21], Lemma 6). This guarantees the existence of sequences of admissible contractions as described below.

Case 1: $\partial(T)=K_{1}$. There exists a sequences of admissible contractions (of vertices from $K_{2}$ ) which reduces $L$ to $H(3)$. By Theorems 1.1, 2.1 and 2.2, $L$ does not embed in $\mathrm{R}^{4}$. 
Case 2: $\partial(T) \neq K_{1}$. Hence $\partial(T) \subseteq K_{2}$ and separates $K_{2}$ into two disks. By performing admissible contractions of pairs of vertices within each of these disks, and within $K_{1}$, we can reduce $L$ to the 2-skeleton of the join $L_{1} * L_{2}$ where $L_{1}$ is the boundary of a triangle and $L_{2}$ is two boundaries of tetrahedra glued along a triangle. Let $v$ be a vertex which belongs to exactly one of the two tetrahedra which were used to define $L_{2}$. Deleting $v$ from $L$ results in $H$ (3) minus one triangle which consists of the vertices of $L_{1}$. Hence the subcomplex $L^{\prime}=(L-v) \cup\left(L_{1} *\{v\}\right)$ of $L$ is admissibly contracted into $H(3)$ by contracting an edge which contains $v$. Thus, $H(3)<L$ and by Theorems 1.1, 2.1 and 2.2, $L$ does not embed in $R^{4}$.

Example 3.6 is a special case of the following conjecture.

CONJECTURe 3.7. Let $K$ be a triangulated $2 d$-sphere and let $T$ be a missing $d$-face in $K$. Let $L=\operatorname{skel}_{d}(K) \cup\{T\}$. Then $L$ does not embed in $\mathrm{R}^{2 d}$.

AdDED IN Proof. Very recently, Uli Wagner and the author verified the conjecture for piecewise linear spheres ([25]).

\section{The obstruction over $Z$}

More commonly in the literature, Van Kampen's obstruction is defined via deleted products and with $Z$ coefficients, where, except for 2-simplicial complexes, its vanishing is also sufficient for embedding of the complex in a Euclidean space of double its dimension. We obtain an analogue of Theorem 1.1 for this context.

The presentation of the background on the obstruction here is based on the ones in [13], [22] and [18].

Let $K$ be a finite simplicial complex. Its deleted product is $K \times K \backslash\{(x, x)$ : $x \in K$, employed with a fixed-point free $\mathbf{Z}_{2}$-action $\tau(x, y)=(y, x)$. It $\mathbf{Z}_{2^{-}}$ deformation retracts into $K_{\times}=\cup\{S \times T: S, T \in K, S \cap T=\emptyset\}$, with which we associate a cell chain complex over Z: $C_{\bullet}\left(K_{\times}\right)=\bigoplus\{\mathrm{Z}(S \times T): S \times T \in$ $\left.K_{\times}\right\}$with a boundary map $\partial(S \times T)=\partial S \times T+(-1)^{\operatorname{dim} S} S \times \partial T$, where $S \times T$ is a $\operatorname{dim}(S \times T)$-chain. The dual cochain complex consists of the $j$-cochains $C^{j}\left(K_{\times}\right)=\operatorname{Hom}_{\mathrm{Z}}\left(C_{j}\left(K_{\times}\right), \mathrm{Z}\right)$ for every $j$.

There is a $Z_{2}$-action on $C_{\bullet}\left(K_{\times}\right)$defined by $\tau(S \times T)=(-1)^{\operatorname{dim}(S) \operatorname{dim}(T)} T \times$ $S$, inducing a $Z_{2}$-action on $C^{\bullet}\left(K_{\times}\right)$. As it commutes with the coboundary map, by restriction of the coboundary map we obtain the subcomplexes of symmetric cochains $C_{s}^{\bullet}\left(K_{\times}\right)=\left\{c \in C^{\bullet}\left(K_{\times}\right): \tau(c)=c\right\}$ and of antisymmetric cochains $C_{a}^{\bullet}\left(K_{\times}\right)=\left\{c \in C^{\bullet}\left(K_{\times}\right): \tau(c)=-c\right\}$. Their cohomology rings are denoted by $H_{s}^{\bullet}\left(K_{\times}\right)$and $H_{a}^{\bullet}\left(K_{\times}\right)$respectively. Let $H_{\mathrm{eq}}^{m}$ be $H_{s}^{m}$ for $m$ even and $H_{a}^{m}$ for $m$ odd. 
For every finite simplicial complex $K$ there is a unique $Z_{2}$-map, up to $Z_{2}$ homotopy, into the infinite dimensional sphere $i: K_{\times} \rightarrow S^{\infty}$, and hence a uniquely defined map $i^{*}: H_{\mathrm{eq}}^{\bullet}\left(S^{\infty}\right) \rightarrow H_{\mathrm{eq}}^{\bullet}\left(K_{\times}\right)$. For $z$ a generator of $H_{\mathrm{eq}}^{m}\left(S^{\infty}\right)$ call $o^{m}=o_{\mathrm{Z}}^{m}\left(K_{\times}\right)=i^{*}(z)$ the Van Kampen obstruction; it is uniquely defined up to a sign. It turns out to have the following explicit description: fix a total order $<$ on the vertices of $K$. It evaluates elementary symmetric chains of even dimension $2 m$ by

$$
o^{2 m}((1+\tau)(S \times T))= \begin{cases}1 & \text { if the unordered pair }\{S, T\} \text { is of } \\ & \text { the form } s_{0}<t_{0}<\cdots<s_{m}<t_{m} \\ 0 & \text { for other pairs }\{S, T\}\end{cases}
$$

and evaluates elementary antisymmetric chains of odd dimension $2 m+1$ by

$$
o^{2 m+1}((1-\tau)(S \times T))= \begin{cases}1 & \text { if }\{S, T\} \text { is of the form } \\ & t_{0}<s_{0}<t_{1}<\cdots<t_{m}<s_{m}<t_{m+1} \\ 0 & \text { for other pairs }\{S, T\}\end{cases}
$$

where the $s_{l}$ 's are elements of $S$ and the $t_{l}$ 's are elements of $T$. Its importance to embeddability is given in the following classical result:

Theorem 4.1. [19], [16], [22] If a simplicial complex $K$ embeds in $\mathrm{R}^{m}$ then $H_{\mathrm{eq}}^{\bullet}\left(K_{\times}\right) \ni o_{\mathrm{Z}}^{m}\left(K_{\times}\right)=0$. If $K$ is $m$-dimensional and $m \neq 2$ then $o_{\mathrm{Z}}^{2 m}\left(K_{\times}\right)=0$ implies that $K$ embeds in $\mathrm{R}^{2 m}$.

In relation to higher minors, the analogue of Theorem 1.1 holds:

Theorem 4.2. Let $H$ and $K$ be simplicial complexes. If $H<K$ and $o_{\mathrm{Z}}^{m}\left(H_{\times}\right) \neq 0$ then $o_{\mathrm{Z}}^{m}\left(K_{\times}\right) \neq 0$.

From Theorems 4.2 and 4.1 it follows that Conjecture 1.3 is true when $2 \operatorname{dim}(H)=m \neq 4$ (and, trivially, when $2 \operatorname{dim}(H)<m)$.

Proof of Theorem 4.2. Fix a total order on the vertices of $K, v_{0}<$ $v_{1}<\cdots<v_{n}$ and consider an admissible contraction $K \mapsto K^{\prime}$ where $K^{\prime}$ is obtained from $K$ by identifying $v_{0} \mapsto v_{1}$ (shortly this will be shown to be without loss of generality). Define a map $\phi$ as follows: for $F \in K^{\prime}$

$$
\phi(F)= \begin{cases}F & \text { if } F \in K \\ \sum\left\{\operatorname{sgn}(v, F)(F \backslash v) \cup v_{0}: v \in F,(F \backslash v) \cup v_{0} \in K\right\} & \text { if } F \notin K\end{cases}
$$

where $\operatorname{sgn}(v, F)=(-1)^{|\{t \in F: t<v\}|}$. Extend linearly to obtain an injective Zchain map $\phi: C_{\bullet}\left(K^{\prime}\right) \longrightarrow C_{\bullet}(K)$. (The check that this map is indeed an injective Z-chain map is similar to the proof of Lemma 3.1.) In case we contract a general $a \mapsto b$, for the signs to work out consider the map $\tilde{\phi}=\pi^{-1} \phi \pi$ 
rather than $\phi$, where $\pi$ is induced by a permutation on the vertices which maps $\pi(a)=v_{0}, \pi(b)=v_{1}$. Then $\tilde{\phi}$ is an injective Z-chain map.

As $\phi(S \times T):=\phi(S) \times \phi(T)$ commutes with the $\mathrm{Z}_{2}$ action and with the boundary map on the chain complex of the deleted product, $\phi$ induces a map $H_{\mathrm{eq}}^{\bullet}\left(K_{\times}\right) \rightarrow H_{\mathrm{eq}}^{\bullet}\left(K_{\times}^{\prime}\right)$. It satisfies $\phi^{*}\left(o_{\mathrm{Z}}^{m}\left(K_{\times}\right)\right)=o_{\mathrm{Z}}^{m}\left(K_{\times}^{\prime}\right)$ for all $m \geq 1$. The checks are straightforward (for proving the last statement, choose a total order with contraction which identifies the minimal two elements $v_{0} \mapsto v_{1}$, and show equality on the level of cochains). We omit the details.

If $K \mapsto K^{\prime}$ is a deletion, consider the injection $\phi: K^{\prime} \rightarrow K$ to obtain again an induced map with $\phi^{*}\left(o_{\mathrm{Z}}^{m}\left(K_{\times}\right)\right)=o_{\mathrm{Z}}^{m}\left(K_{\times}^{\prime}\right)$.

Let the sequence $K=K^{0} \mapsto K^{1} \mapsto \cdots \mapsto K^{t}=H$ demonstrate the fact that $H<K$. By composing the corresponding maps as above we obtain a map $\phi^{*}$ with $\phi^{*}\left(o_{\mathrm{Z}}^{m}\left(K_{\times}\right)\right)=o_{\mathrm{Z}}^{m}\left(H_{\times}\right)$and the result follows.

\section{Topology preserving edge contractions}

\subsection{PL manifolds}

Proof OF Theorem 1.4. Let $M$ be a PL-triangulation of a compact $d$-manifold without boundary. Let $a b$ be an edge of $M$ and let $M^{\prime}$ be obtained from $M$ by contracting $a \mapsto b$. We will prove that if the Link Condition (2) holds for $a b$ then $M$ and $M^{\prime}$ are PL-homeomorphic, and otherwise they are not homeomorphic (not even 'locally homologic'). For $d=1$ the assertion is clear. Assume $d>1$. Denote the closed star of a vertex $a$ in $M$ by st $(a, M)=\{T \in$ $M: T \cup\{a\} \in M\}$ and denote its antistar by ast $(a, M)=\{T \in M: a \notin T\}$.

Denote $B(b)=\{b\} *$ ast $(b, \operatorname{lk}(a, M))$ and $L=\operatorname{ast}(a, M) \cap B(b)$. Then $M^{\prime}=\operatorname{ast}(a, M) \cup_{L} B(b)$. As $M$ is a PL-manifold without boundary, $\operatorname{lk}(a, M)$ is a $(d-1)$-PL-sphere (see e.g. Corollary 1.16 in [6]). By Newman's theorem (e.g. [6], Theorem 1.26) ast $(b, \mathrm{k}(a, M))$ is a $(d-1)$-PL-ball. Thus $B(b)$ is a $d$-PL-ball. Observe that $\partial(B(b))=\operatorname{ast}(b, \operatorname{lk}(a, M)) \cup\{b\} * \operatorname{lk}(b, \operatorname{lk}(a, M))=$ $\operatorname{lk}(a, M)=\partial(\operatorname{st}(a, M))$.

The identity map on $\operatorname{lk}(a, M)$ is a PL-homeomorphism $h: \partial(B(b)) \rightarrow$ $\partial(\operatorname{st}(a, M))$, hence it extends to a PL-homeomorphism $\tilde{h}: B(b) \rightarrow \operatorname{st}(a, M)$ (see e.g. [6], Lemma 1.21).

Note that $L=\operatorname{lk}(a, M) \cup(\{b\} *(\operatorname{lk}(a, M) \cap \operatorname{lk}(b, M)))$.

If $\operatorname{lk}(a) \cap 1 \mathrm{k}(b)=\operatorname{lk}(a b)$ (in $M$ ) then $L=\operatorname{lk}(a, M)$, hence gluing together the maps $\tilde{h}$ and the identity map on ast $(a, M)$ results in a PL-homeomorphism from $M^{\prime}$ to $M$.

If $\operatorname{lk}(a) \cap \operatorname{lk}(b) \neq 1 \mathrm{k}(a b)$ (in $M$ ) then $\operatorname{lk}(a, M) \varsubsetneqq L$. The case $L=B(b)$ implies that $M^{\prime}=\operatorname{ast}(a, M)$ and hence $M^{\prime}$ has a nonempty boundary, showing it is not homeomorphic to $M$. A small punctured neighborhood of a point in the boundary of $M^{\prime}$ has trivial homology while all small punctured neighborhoods 
of points in $M$ has non vanishing $(d-1)$-th homology. This is what we mean by 'not even locally homologic': $M$ and $M^{\prime}$ have homologically different sets of small punctured neighborhoods.

We are left to deal with the case $\operatorname{lk}(a, M) \varsubsetneqq L \varsubsetneqq B(b)$. As $L$ is closed there exists a point $t \in L \cap \operatorname{int}(B(b))$ with a small punctured neighborhood $N\left(t, M^{\prime}\right)$ which is not contained in $L$. For a subspace $K$ of $M^{\prime}$ denote by $N(t, K)$ the neighborhood in $K N\left(t, M^{\prime}\right) \cap K$. Thus $N\left(t, M^{\prime}\right)=N(t$, ast $(a, M)) \cup_{N(t, L)}$ $N(t, B(b))$. We get a Mayer-Vietoris exact sequence in reduced homology:

$$
\begin{aligned}
H_{d-1} N(t, L) & \longrightarrow H_{d-1} N(t, \operatorname{ast}(a, M)) \oplus H_{d-1} N(t, B(b)) \\
& \longrightarrow H_{d-1} N\left(t, M^{\prime}\right) \longrightarrow H_{d-2} N(t, L) \\
& \longrightarrow H_{d-2} N(t, \operatorname{ast}(a, M)) \oplus H_{d-2} N(t, B(b))
\end{aligned}
$$

Note that $N(t$ ast $(a, M))$ and $N(t, B(b))$ are homotopic to their boundaries which are $(d-1)$-spheres. Note further that $N(t, L)$ is homotopic to a proper subset $X$ of $\partial(N(t, B(b)))$ such that the pair $(\partial(N(t, B(b))), X)$ is triangulated. By Alexander duality $H_{d-1} N(t, L)=0$. Thus, (9) simplifies to the exact sequence

$$
0 \longrightarrow \mathrm{Z} \oplus \mathrm{Z} \longrightarrow H_{d-1} N\left(t, M^{\prime}\right) \longrightarrow H_{d-2} N(t, L) \longrightarrow 0 .
$$

Thus, $\operatorname{rank}\left(H_{d-1} N\left(t, M^{\prime}\right)\right) \geq 2$, hence $M$ and $M^{\prime}$ are not locally homologic, and in particular are not homeomorphic.

Remarks. (1) Omitting the assumption in Theorem 1.4 that the boundary is empty makes both implications incorrect. Contracting an edge to a point shows that the Link Condition is not sufficient. Contracting an edge on the boundary of a cone over an empty triangle shows that the Link Condition is not necessary.

(2) The necessity of the Link Condition holds also in the topological category (and not only in the PL category), as the proof of Theorem 1.4 shows. Indeed, for this part we only used the fact that $B(b)$ is a pseudo manifold with boundary $\operatorname{lk}(a, M)$ (not that it is a ball); taking the point $t$ to belong to exactly two facets of $B(b)$. The following part, in the topological category, is still open:

Problem 5.1. Given an edge in a triangulation of a compact manifold without boundary which satisfies the Link Condition, is it true that its contraction results in a homeomorphic space? Or at least in a space of the same homotopic or homological type?

A Mayer-Vietoris argument shows that such topological manifolds $M$ and $M^{\prime}$ have the same Betti numbers; both st $(a, M)$ and $B(b)$ are cones and hence their reduced homology vanishes. 
A candidate for a counterexample for Problem 5.1 may be the join $M=$ $T * P$ where $T$ is the boundary of a triangle and $P$ a triangulation of Poincaré homology 3-sphere, where an edge with one vertex in $T$ and the other in $P$ satisfies the Link Condition. By the double-suspension theorem (Edwards [3] and Cannon [1]) $M$ is a topological 5-sphere.

Walkup [20] mentioned, without details, the necessity of the Link Condition for contractions in topological manifolds, as well as the sufficiency of the Link Condition for the 3 dimensional case (where the category of PL-manifolds coincides with the topological one); see [20], p. 82-83.

\subsection{PL spheres}

In this section we use some terminology from $f$-vectors theory; readers unfamiliar with this terminology can consult [17].

Definition 5.2. Boundary complexes of simplices are strongly edge decomposable and, recursively, a triangulated PL-manifold without boundary $S$ is strongly edge decomposable if it has an edge which satisfies the Link Condition (2) such that both its link and its contraction are strongly edge decomposable.

By Theorem 1.4 the complexes in Definition 5.2 are all triangulated PLspheres. Note that every 2 -sphere is strongly edge decomposable.

Let $v u$ be an edge in a simplicial complex $K$ which satisfies the Link Condition, whose contraction $u \mapsto v$ results in the simplicial complex $K^{\prime}$. Note that the $f$-polynomials satisfy

$$
f(K, t)=f\left(K^{\prime}, t\right)+t(1+t) f(\operatorname{lk}(\{v u\}, K), t),
$$

hence the $h$-polynomials satisfy

$$
h(K, t)=h\left(K^{\prime}, t\right)+t h(\operatorname{lk}(\{v u\}, K), t) .
$$

We conclude the following:

COROLlaRY 5.3. The g-vector of strongly edge decomposable triangulated spheres is non negative.

Is it also an $M$-sequence? The strongly edge decomposable spheres (strictly) include the family of triangulated spheres which can be obtained from the boundary of a simplex by repeated Stellar subdivisions (at any face); the later are polytopal, hence their $g$-vector is an $M$-sequence. For the case of subdividing only at edges (10) was considered by Gal ([5], Proposition 2.4.3). 


\section{Graph minors versus higher minors}

While Theorem 1.1 is an instance of a property of graph minors which generalizes to higher minors, this is not always the case. Let us mention some properties which do not generalize, and others for which we do not know whether they generalize or not.

- For graphs, if $K$ is a subdivision of $H$ then $H$ is a minor of $K$. This is not the case for higher minors.

ExAmPLe 6.1. Let $H$ be a triangulated PL 3-sphere whose triangulation contains a knotted triangle $\{12,23,13\}$ (e.g. [10] for an example with few vertices and references to Hachimori's first examples. In [7] such spheres were proved to be non-constructible). Then $H$ is a subdivision of $\partial \Delta^{4}$, the boundary complex of the 4-simplex, but $\partial \Delta^{4}$ is not a minor of $H$.

Proof. Consider, by contradiction, a sequence of deletions and admissible contractions starting at $H$ and ending at $\partial \Delta^{4}$. Any deletion would result in a complex with a vanishing 3-homology; further deletions and contractions would keep the 3-homology being zero as they induce the injective chain map from Theorem 3.4. Thus the sequence contains only contractions. Any admissible contraction, assuming we haven't reached $\partial \Delta^{4}$ yet, must satisfy the Link Condition (2) - as by Alexander duality a sphere can not contain a sphere of the same dimension as a proper subspace. If a contraction $a \mapsto b$ satisfies $a \neq 1,2,3$, the PL-homeomorphism constructed in the proof of Theorem 1.4 shows that it results in a PL 3 -sphere with $\{12,23,13\}$ a knotted triangle. It suffices to show that a contraction where $a \in\{1,2,3\}$ also results in a triangulation with a knotted triangle, as this would imply that $\partial \Delta^{4}$ can never be reached, a contradiction. Without loss of generality $a=1$. Let $M^{\prime}$ be obtained from $M$ by contract: $a \mapsto b$ as above.

As $\{12,23,13\}$ is knotted in $M$, the Link Condition implies $b \neq 2,3$ and $\{b, 2,3\} \notin M$. Thus $\{b 2,23, b 3\}$ is an induced subcomplex in $M^{\prime}$, and hence there is a deformation retract of $M^{\prime}-\{b 2,23, b 3\}$ onto the induced subcomplex $M^{\prime}\left[V\left(M^{\prime}\right)-\{b, 2,3\}\right]=M[V(M)-\{b, 1,2,3\}]$, where $V(K)$ is the set of vertices of a complex $K$ (e.g. [24], Lemma 70.1). Similarly, $M[V(M)-\{b, 1,2,3\}]$ is a deformation retract of $M[V(M)-\{1,2,3\}]-\{b\}$. To show that the fundamental group $\pi_{1}\left(M^{\prime}-\{b 2,23, b 3\}\right) \neq 0$ we will show that $\pi_{1}(M[V(M)-\{1,2,3\}]-\{b\}) \neq 0$. We use Van Kampen's theorem for the union $M[V(M)-\{1,2,3\}]=(M[V(M)-\{1,2,3\}]-\{b\}) \cup$ $\operatorname{int}(\operatorname{star}(b, M[V(M)-\{1,2,3\}]))$ : note that the intersection is a deformation retract of $l k(b, M)$ minus the induced subcomplex on $\{1,2,3\}$ in it, which is path-connected and simply connected. We conclude that $\pi_{1}(M[V(M)-$ $\{1,2,3\}]-\{b\}) \cong \pi_{1}(M[V(M)-\{1,2,3\}]) \neq 0$, as $\{12,23,13\}$ is knotted 
in $M$.

- For a graph $K$ on $n$ vertices, if $K$ has more than $3 n-6$ edges then it contains a $K_{5}$ minor (Mader proved that it even contains a $K_{5}$ subdivision [11]). Is the following generalization to higher minors true?:

Problem 6.2. Let $C(d, n))$ be the boundary complex of a cyclic d-polytope on $n$ vertices, and let $K$ be a simplicial complex on $n$ vertices. Does $f_{d}(K)>$ $f_{d}(C(2 d+1, n))$ imply $H(d+1)<K$ ?

EXAmPLE 6.3. Let $M_{L}$ be the vertex transitive neighborly 4-sphere on 15 vertices manifold_ $(4,15,5,1)$ found by Frank Lutz [9].

$M_{L}$ has no universal edges, i.e., every edge is contained in a missing triangle.

It is possible that $K$ equals the 2-skeleton of $M_{L}$ union with a missing triangle would provide a counterexample to Problem 6.2.

- If $K$ is the graph of a triangulated 2-sphere union with a missing edge then it contains a $K_{5}$ minor (the condition implies having more than $3 n-6$ edges). Is the following generalization to higher minors true?:

Problem 6.4. Let $K$ be the union of the $d$-skeleton of a triangulated $2 d$ sphere with a missing $d$-face. Does $H(d+1)<K$ ?

It is possible that $K$ equals the onion of the 2-skeleton of $M_{L}$ with a missing triangle would provide a counterexample. But if true, then by Theorems 1.1, 2.1 and 2.2, Conjecture 3.7 will follow.

- A Robertson-Seymour type theorem does not hold for embeddability in higher dimensional spheres:

Proposition 6.5. For any $d \geq 2$ There exist infinitely many $d$-complexes not embeddable in the $2 d$-sphere such all of their proper minors do embed in the $2 d$-sphere.

Proof. By identifying disjoint pair of points, each pair to a point, where each pair lies in the interior of a facet of $H(d+1)$, one obtains topological spaces which are not embeddable in the $2 d$-sphere but such that any proper subspace of them is. This was proved by Zaks [23] for $d>2$ and later by Ummel [18] for $d=2$. By choosing say $m$ such pairs in each facet, one obtains infinitely many pairwise non-homeomorphic such spaces when $m$ varies. To conclude the claim it suffices to triangulate these spaces in a way that no contraction would be admissible; this is indeed possible (see Figure 1 for an illustration): first subdivide each facet into $m$ small facets say. To identify simplicialy a pair of points $s, t$ in the interior of a small facet $F=\left\{v_{0}, \ldots, v_{d}\right\}$ first further subdivide $F$ as follows. Consider the prism 




FIGURE 1. Subdivision of a small facet $F=\left\{v_{0}, v_{1}, v_{2}\right\}$.

$[0,1] \times\left\{v_{1}, \ldots, v_{d}\right\}$ with bottom $\left\{v_{1}, \ldots, v_{d}\right\}$ and top $\left\{v_{1}^{\prime}, \ldots, v_{d}^{\prime}\right\}$ and triangulate the cylinder $[0,1] \times \partial\left\{v_{1}, \ldots, v_{d}\right\}$ without adding new vertices (this is standard). Now cone with a vertex $v_{0}^{\prime}$ over $\partial\left([0,1] \times\left\{v_{1}, \ldots, v_{d}\right\}\right)$ to obtain a triangulation of the prism, and further cone with the vertex $v_{0}$ over $\partial\left([0,1] \times\left\{v_{1}, \ldots, v_{d}\right\}\right)-\left\{v_{1}, \ldots, v_{d}\right\}$ to obtain, together with the prism, a triangulation of $F$. Subdivide $\left\{v_{1}, \ldots, v_{d}, v_{0}^{\prime}\right\}$ by staring from a vertex $s$ in its interior, and subdivide $\left\{v_{1}^{\prime}, \ldots, v_{d}^{\prime}, v_{0}\right\}$ by staring from a vertex $t$ in its interior. Note that identifying $s \mapsto t$ results in a complex where each pair of vertices from $v_{0}, \ldots, v_{d}, v_{0}^{\prime}, \ldots, v_{d}^{\prime}, t$ is contained in a missing face of dimension $<d$ (a facet for a pair from $v_{0}, \ldots, v_{d}$ or from $v_{0}^{\prime}, \ldots, v_{d}^{\prime}$, and an edge or a triangle with the vertex $t$ for the rest of the pairs).

ACKNOWLEDGEMENTS. I wish to thank Gil Kalai and Eric Babson for helpful discussions, and Uli Wagner and Karanbir Sarkaria for helpful remarks on the presentation. Part of this work was done during the author's stay at Institut Mittag-Leffler, supported by the ACE network. The author thanks the organizers of a special semester there, Anders Björner and Richard Stanley, and Institut Mittag-Leffler, for their hospitality.

\section{REFERENCES}

1. Cannon, J. W., Shrinking cell-like decompositions of manifolds. Codimension three, Ann. of Math. 110 (1979), 83-112. 
2. Dey, T. K., Edelsbrunner, H., Guha, S., and Nekhayev, D. V., Topology preserving edge contraction, Publ. Inst. Math. (Beograd) (N.S.) 66(80) (1999), 23-45.

3. Edwards, R. D., The double suspension of a certain homology 3-sphere is $S^{5}$, Notices Amer. Math. Soc. 22 (1975), A-334.

4. Flores, A., Über $n$-dimensionale Komplexe die im $R^{2 n+1}$ absolut selbstverschlungen sind, Ergeb. Math. Kolloq. 6 (1933/34), 4-7.

5. Gal, Ś. R., Real root conjecture fails for five- and higher-dimensional spheres, Discrete Comput. Geom. 34 (2005), 269-284.

6. Hudson, J. F. P., Piecewise-linear Topology, Benjamin Inc., New York 1969.

7. Hachimori M., and Ziegler, G. M., Decompositions of simplicial balls and spheres with knots consisting of few edges, Math. Z. 235 (2000), 159-171.

8. Kuratowski, K., Sur le probléme des courbes gauches en topologie, Fund. Math. 15 (1930), 271-283.

9. Lutz, F. H., http://www.math.tu-berlin.de/diskregeom/stellar/.

10. Lutz, F. H., Small examples of non-constructible simplicial balls and spheres, SIAM J. Discrete Math. 18 (2004), 103-109.

11. Mader, W., $3 n-5$ edges do force a subdivision of $K_{5}$, Combinatorica 18 no. 4 (1998), 569-595.

12. Matoušek, J., Using the Borsuk-Ulam Theorem, Springer-Verlag, Berlin Heidelberg, 2003.

13. Novik, I., A note on geometric embeddings of simplicial complexes in a Euclidean space, Discrete Comput. Geom. 23 (2000), 293-302.

14. Sarkaria, K. S., Shifting and embeddability of simplicial complexes, a talk given at Max-Planck Institut für Math., Bonn, MPI 92-51 (1992).

15. Sarkaria, K. S., Shifting and embeddability, unpublished manuscript (1992).

16. Shapiro, A., Obstructions to the embedding of a complex in a Euclidean space, I. the first obstruction, Ann. of Math. 66 (1957), 256-269.

17. Stanley, R. P., Combinatorics and Commutative Algebra, Prog. Math. 41 (1983).

18. Ummel, B. R., Imbedding classes and n-minimal complexes, Proc. Amer. Math. Soc. 38 (1973), 201-206.

19. Van Kampen, E. R., Komplexe in euklidischen Räumen, Abh. Math. Sem. 9 (1932), 72-78.

20. Walkup, D. W., The lower bound conjecture for 3- and 4-manifolds, Acta Math. 125 (1970), 75-107.

21. Whitely, W., Vertex splitting in isostatic frameworks, Structural Topology 16 (1989), 23-30.

22. Wu, T. W., A Theory of Imbedding, Immersion and Isotopy of Polytopes in a Euclidean Space, Science Press, Peking, 1965.

23. Zaks, J., On minimal complexes, Pacific J. Math. 28 (1969), 721-727.

24. Munkres, J. R., Elements of Algebraic Topology, Addison-Wesley Publishing Company, Menlo Park, CA, 1984.

25. Nevo, E., Wagner, U., On the embeddability of skeleta of spheres, math arXiv:0709.0988, submitted, 2007.

INSTITUTE OF MATHEMATICS

HEBREW UNIVERSITY

JERUSALEM

ISRAEL

E-mail: eranevo@math.huji.ac.il 